\title{
Ansiedade entre gestantes atendidas na atenção básica
}

\author{
Anxiety among pregnant women assisted in primary care \\ Ansiedad entre mujeres embarazadas cuidadas con atención básica
}

Rayane Bezerra da Silva ORCID: https://orcid.org/0000-0001-8666-2803 Faculdade de Enfermagem Nova Esperança. Brasil E-mail: rayanebt123@gmail.com

Viviane Cordeiro de Queiroz ORCID: https://orcid.org/0000-0002-2037-921X Universidade Federal da Paraíba, Brasil

E-mail: vivicordeiroqueiroz35@gmail.com

Smalyanna Sgren da Costa Andrade ORCID: https://orcid.org/0000-0002-9812-9376

Faculdade de Enfermagem Nova Esperança. Brasil E-mail: smalyanna@hotmail.com

Edna Samara Ribeiro César ORCID: https://orcid.org/0000-0002-1150-5157 Faculdade de Enfermagem Nova Esperança. Brasil E-mail: samaraenfermagem@outlook.com

Vilma Felipe Costa de Melo ORCID: https://orcid.org/0000-0001-5721-3240 Faculdade de Enfermagem Nova Esperança. Brasil E-mail: vilmelopsi@yahoo.com.br

Simone Helena dos Santos Oliveira

ORCID: https://orcid.org/0000-0002-9556-1403 Universidade Federal da Paraíba, Brasil E-mail: simonehsoliveira@gmail.com

\begin{abstract}
Resumo
Objetivo: verificar a relação entre manifestações clínicas e aspectos sociais, reprodutivos e relacionados à gestação de mulheres e elaborar diagnósticos de enfermagem. Método: trata-se de pesquisa transversal, realizada com 30 gestantes durante o pré-natal em município nordestino por meio do State - Trait Anxiety Inventory e formulário de manifestações clínicas do transtorno da ansiedade generalizada, com análise descritiva e inferencial. Resultados: Houve relações estatísticas entre número de consulta pré-natal e nervosismo, transpiração com etnia e com mulheres com menos de 20 semanas gestacionais. Maior renda foi fator de proteção à tontura e mais de seis consultas pré-natais é fator de proteção ao medo. Conclusão: Atenção especial frente às manifestações clínicas da ansiedade deve ser dada às mulheres não brancas, com idade superior a 26 anos. Os diagnósticos de enfermagem como sono comprometido e padrão de repouso alterado devem ser incorporados no plano de cuidados da atenção básica.
\end{abstract}

Palavras-chave: Ansiedade; Cuidado pré-natal; Diagnóstico de enfermagem; Gestantes; Saúde da família.

\begin{abstract}
Objective: to verify the relationship between clinical manifestations and social, reproductive and pregnancy-related aspects in women and to prepare nursing diagnoses. Method: cross-sectional research with 30 pregnant women during prenatal in a northeastern city using the State - Trait Anxiety Inventory and a form for clinical manifestations of generalized anxiety disorder, with descriptive and inferential analysis. Results: There were statistical relations between number of prenatal visits and nervousness, sweating with ethnicity and with women with less than 20 weeks gestational age. Higher income was protective factor for dizziness and more than six prenatal visits is a protective factor for fear. Conclusion: Special attention to the clinical manifestations of anxiety should be given to non-white women over 26 years of age. Nursing diagnoses such as impaired sleep and altered rest pattern should be incorporated into the primary care plan.
\end{abstract}

Keywords: Anxiety; Prenatal care; Nursing diagnosis; Pregnant women; Family health.

\section{Resumen}

Objetivo: verificar la relación entre las manifestaciones clínicas y los aspectos sociales, reproductivos y relacionados con el embarazo de la mujer y elaborar diagnósticos de enfermería. Método: se trata de investigación transversal con 30 gestantes durante el prenatal de una ciudad del noreste utilizando el Inventario Estado - Rasgo de Ansiedad y una forma de manifestaciones clínicas del trastorno de ansiedad generalizada, con análisis descriptivo e inferencial. 
Resultados: Hubo relaciones estadísticas entre el número de visitas prenatales y el nerviosismo, sudoración con la etnia y con mujeres con menos de 20 semanas de gestación. Los ingresos más altos fueron factor de protección contra el mareo y más de seis consultas prenatales son factores de protección contra el miedo. Conclusión: Se debe prestar especial atención a las manifestaciones clínicas de ansiedad a las mujeres no blancas, mayores de 26 años. Los diagnósticos de enfermería como el sueño comprometido y los patrones de descanso alterados deben incorporarse al plan de atención primaria.

Palabras clave: Ansiedad; Cuidado prenatal; Diagnóstico de enfermeira; Mujeres embarazadas; Salud de la família.

\section{Introdução}

A gestação é um momento de modificações na vida da mulher que traz repercussões física, social e psicológica. Sobre as mudanças psicossociais, essa condição pode trazer oscilação emocional, favorecendo medo e insegurança (Meireles, Neves, Carvalho \& Ferreira, 2017). Por isso, embora seja um momento marcado por alegrias e realizações, a gestante pode vivenciar situações de ansiedade (Silva, Nogueira, Clapis \& Leite, 2017).

O número de mulheres com transtornos mentais permite avaliar a magnitude do problema de maneira global. Segundo a Organização Mundial da Saúde, cerca de 450 milhões de mulheres e homens sofrem de algum transtorno, responsável por $8,8 \%$ da mortalidade e $16,6 \%$ das morbidades associadas. Resultados mostram que as mulheres apresentam maiores prevalências em relação aos homens, quanto aos transtornos depressivo, somatoformes e de ansiedade (Organização Mundial da Saúde, 2017).

Para tanto, a ansiedade na gestação pode ser desenvolvida devido aos pensamentos negativos sobre mudança corporal, provocando baixa autoestima. Esses estímulos podem acontecer em decorrência das preocupações, nervosismos e apreensão, ocasionando consequências à gestante e ao bebê (Meireles, Neves, Carvalho \& Ferreira, 2017). Sobre isso, estudo longitudinal afirmou que a gestação e o puerpério são reconhecidos como fatores de risco para o desenvolvimento e exacerbação de adoecimentos mentais (Costa, Souza, Pedroso \& Strufaldi, 2018).

Com efeito, transtornos de ansiedade configuram-se fatores de risco para fragilidades no desenvolvimento fetal e complicações obstétricas, além de efeitos danosos futuros no desenvolvimento físico e psicológico do bebê (Silva, Nogueira, Clapis \& Leite, 2017). Nesse contexto, a assistência de enfermagem satisfatória durante o pré-natal favorece a qualidade dos serviços e as práticas de saúde no campo materno-infantil, contribuindo para desfechos perinatais favoráveis (Nunes, Gomes, Rodrigues \& Mascarenhas, 2016).

Para tanto, este estudo se justifica pela importância de enaltecer a identificação dos sintomas de ansiedade na gestação, por meio da utilização de ferramentas em saúde mental enquanto recurso salutar direcionado às mulheres atendidas em unidades de saúde como parte do processo de enfermagem, o que pode favorecer a categoria no que tange à incorporação de saberes de diversas áreas que favoreçam o cuidado sistematizado. Assim, este estudo foi norteado pelo seguinte questionamento: qual a relação da ansiedade com a caracterização das gestantes atendidas na atenção básica? Para tanto, objetivou-se verificar a relação entre manifestações clínicas e aspectos sociais, reprodutivos e relacionados à gestação de mulheres e elaborar diagnósticos de enfermagem.

\section{Metodologia}

Pesquisa transversal e abordagem quantitativa desenvolvida em todas as quatro Unidades de Saúde da Família da zona urbana da Baía da Traição, Paraíba, com as gestantes atendidas durante o pré-natal, conforme os seguintes critérios: maioridade etária, gestantes cadastradas para atendimento pré-natal, com qualquer grau de escolaridade.

Os critérios de exclusão foram: gestantes não aptas emocionalmente (autorreferido) e diagnosticadas com transtorno mental (autorreferido) para não causar viés nos resultados, considerando que a finalidade do estudo não foi repetir um diagnóstico médico, mas identificar mulheres aparentemente saudáveis que estavam com sintomas ansiosos, por meio de 
ferramentas científicas que a Enfermagem pode fazer uso durante o pré-natal, melhorando a anamnese para elaboração de um plano de cuidados, a partir dos Diagnósticos de Enfermagem.

A amostra por conveniência considerou a Teoria Central do Limite do âmbito da estatística que justifica o número mínimo de 30 participantes para tratamentos analíticas (Triola, 2017). A pesquisa foi realizada conforme o funcionamento do atendimento pré-natal, no turno da manhã, durante o mês de agosto e setembro de 2019, em salas reservadas nos serviços. Os resultados foram elaborados com base em quatro etapas.

A primeira etapa foi a coleta dos dados por meio do formulário de caracterização composto por dados sociodemográficos (idade, anos de escolarização, rendimento salarial, naturalidade, religião, etnia, ocupação) sexual e reprodutivos (sexarca, situação conjugal, paridade, abortamento, planejamento da gravidez, semanas gestacionais), acompanhamento pré-natal (número de consultas) e hábitos de vida (etilismo, tabagismo e sedentarismo).

Subsequentemente, na segunda etapa aplicou-se o Inventário de Ansiedade Traço-Estado, do inglês, The State - Trait Anxiety Inventory (STAI) retrata uma escala que faz uma avaliação do grau de ansiedade enquanto ansiedade-estado, isto é, state (STAI-S) caracterizado como momento emocional transitório, com tensão e sensação desagradáveis conscientemente percebidos; e outra que analisa a ansiedade enquanto ansiedade-traço, ou seja, trait (STI-T), que se refere a um aspecto mais estável em que o indivíduo sabe lidar com a situação da ansiedade durante a vida, perpassando pelo diagnóstico. O STI-S é uma escala traduzida e validada no Brasil e tem sido amplamente utilizada na Enfermagem em estudos de natureza semelhante (Biaggio \& Natalício, 2003).

O STI-S é um instrumento de autoavaliação, composto por 20 itens, do tipo Likert variam de absolutamente não (1), um pouco (2), bastante (3) e muitíssimo (4). O escore total para o somatório dos 20 itens para cada participante varia de 20-80 pontos. A classificação adotada para os níveis de ansiedade foram graus: baixo (0-34 pontos), moderado (35-49), alto (50-64) e muito alto (65-80) (Fontoura, Cardoso, Rodrigues, Almeida \& Carvalho, 2018). Vale ressaltar que o estudo não objetivou diagnosticar a ansiedade como transtorno, considerando que esta é uma atribuição médica, mas enquanto variável identificada pela classificação dos graus deste transtorno emergentes nos somatórios de um instrumento multidisciplinar. Embora o STI-S seja um questionário, para esta pesquisa, optou-se por entrevistar individualmente (formulário) cada participante para inserção de mulheres com qualquer grau de escolaridade.

Durante a análise, a pontuação da escala Likert sofreu inversão dos itens 1, 2, 5, 8, 10,11, 15 e 16 para conferir melhor fidedignidade aos itens do instrumento e ao somatório dos estratos conforme a classificação dos graus de ansiedade. Nesses itens, a escala ficou absolutamente não (4), um pouco (3), bastante (2) e muitíssimo (1).

Ao final da aplicação do STI-S, ocorreu a terceira etapa, com uso de outro instrumento criado pelas pesquisadoras com possíveis sintomas da ansiedade generalizada inspirado no protocolo da rede de atenção psicossocial do sistema único de saúde baseado em evidências, para o acolhimento e o tratamento de transtornos de ansiedade generalizada, para livre resposta às manifestações clínicas vivenciadas no processo gestacional.

Por fim, após a coleta dos dados ocorreu a quarta etapa que foi a construção dos diagnósticos de enfermagem, sendo definido que na existência de pelo menos uma participante eliciando alguma manifestação, esta seria considerada como característica definidora para a elaboração dos possíveis diagnósticos de enfermagem para o sintoma. Esse momento ocorreu na intenção de enaltecer o processo de enfermagem, reafirmando a possibilidade de uso de instrumentos multidisciplinares para a elaboração de uma etapa do cuidado da categoria. Os Diagnósticos de Enfermagem foram formulados por meio da CIPE® (Resende et al., 2019), restringindo-se ao foco e ao julgamento, com base na manifestação clínica do transtorno da ansiedade generalizada como característica definidora. Todos os diagnósticos foram consultados quanto à sua descrição, com vistas a manter a coerência com o sinal/sintoma eliciado pela gestante.

Utilizou-se o teste de Shapiro-Wilk, indicando a normalidade dos dados. A análise foi realizada com o auxílio do 
Programa Statistical Package for the Social Sciences, versão 21, por meio de estatística descritiva e inferencial. Torna-se importante enfatizar que os testes de associação dos dados para variáveis categóricas foram o Qui-quadrado (cruzamentos com frequência $\geq 5$ gestantes nas caselas) e o teste exato de Fisher foi utilizado para as caselas com frequência absoluta menor que cinco, ambos com significância $p \leq 0,05$. A razão de chance e intervalo de confiança indica a chance ou a proteção determinada pelas variáveis (valores significativos não perpassam pela nulidade simbolizada pelo número 1).

Embora haja outras formas de leitura do odds ratio, neste estudo, o fator de proteção, foi subtraído do valor de 1, indicando a menor chance em percentual. As hipóteses foram: existe relação entre aspectos sociais, reprodutivos e relacionados à gestação com a classificação da ansiedade conforme o STI-S, bem como com as manifestações clínicas do transtorno da ansiedade generalizada. O Termo de Consentimento Livre e Esclarecido (TCLE) foi assinado pelos participantes da pesquisa em duas vias. A pesquisa obedeceu aos preceitos éticos da pesquisa envolvendo seres humanos em conformidade com a resolução 466/12 do CNS, sendo aprovada pelo Comitê de Ética da Faculdade de Enfermagem Nova Esperança, conforme número do Parecer 3.389.105/2019 e CAAE n.15286619.2.0000.5179 em 13/06/2019.

\section{Resultados}

A idade média foi de $28,40(\mathrm{DP} \pm 6,015)$, com $8(26,5 \%)$ entre $18-25$ anos e $22(73,3 \%)$ idade $\geq 26$ anos, $10(33,3 \%)$ tinham até nove anos de estudo, enquanto $20(66,6 \%)$ tinham $\geq 10$ anos de estudo, $29(96,7 \%)$ viviam com rendimento inferior a dois salários mínimos por mês (um salário equivale a $\mathrm{R} \$ 934,00$ ), enquanto $1(3,3 \%)$ possuía renda $\geq 2$. Além disso, 8 (26,6\%) eram da Baía da Traição, 22 (73,3\%) eram de outros municípios agrupados em única variável, 21 (70\%) eram católicas, 6 (20,0\%) evangélicas e $3(10,0 \%)$ acreditavam em outras crenças religiosas, 12 (40,0\%) se autodeclararam pardas, 6 (20,0\%) negras, $5(16,7 \%)$ brancas, $2(6,6 \%)$ asiáticas, $5(16,7 \%)$ não souberam a sua etnia, $5(16,6 \%)$ eram estudantes e 25 $(83,4 \%)$ exercia ocupação remunerada, sendo profissional liberal, da saúde, educação e funcionárias do setor público (dados não expostos em tabela).

Além disso, a idade média da sexarca foi de 16,6 (DP $\pm 2,99)$, com 15 (50,0\%) para iniciação antes dos 15 anos e igual prevalência para maiores de 16 anos, $27(90,0 \%)$ foram a menos de cinco consultas, $3(10,0 \%)$ frequentaram mais de 6 consultas, $21(70,0 \%)$ não sofreram abortamento, $9(30,0 \%)$ passaram por algum episódio de interrupção da gestação, 22 (73,3\%) eram primigestas, 8 (26,6\%) multigestas, 17 (56,7\%) não planejaram a gestação, 13 (43,3\%) planejaram, $17(56,6 \%)$ estavam com mais de 20 semanas gestacionais e 13 (43,3\%) tinham menos de 20 semanas (dados não expostos em tabela).

Não obstante, 25 (83,3\%) não fumam, 20 (66,6\%) não são etilistas e $5(16,6 \%)$ são sedentárias. Das mulheres que não são sedentárias, 9 (30,0\%) faz exercício esporádico de 1-2 vezes na semana e $16(53,3 \%)$ praticam 2-3 vezes na semana. Sobre a classificação da ansiedade, $3(10,0 \%)$ das mulheres tinham ansiedade baixa, $26(86,7 \%)$ moderada e $1(3,3 \%)$ alta. A associação entre os aspectos sociais, reprodutivos e número de consultas pré-natais com ansiedade, por meio do Teste Exato de Fisher, não apresentaram relação significativa, refutando a primeira hipótese, ou seja, nenhuma característica específica influencia na classificação do transtorno. Mesmo não havendo associação entre as variáveis, é importante ressaltar as maiores proporções de ansiedade moderada para gestantes com idade $\geq 26$ anos $(66,6 \%$; $=0,399)$, com mais de dez anos de escolarização $(53,3 \% ; \mathrm{p}=0,682)$, não-brancas $(73,3 \% ; \mathrm{p}=0,547)$, com rendimento mensal de até um salário mínimo $(60 \%$; $\mathrm{p}=0,178)$, católica $(63,3 \% ; \mathrm{p}=0,204)$, com parceiro $(66,6 \% ; \mathrm{p}=1,000)$, sexarca antes dos 15 anos $(46,6 \% ; \mathrm{p}=0,593)$ e com menos de seis consultas de pré-natal $(80 \% ; \mathrm{p}=0,346)$ (dados não expostos em tabela).

Aspectos sociais, reprodutivos e relacionados à gestação não apresentaram relação significativa, tampouco se configuraram como fatores de risco ou proteção à sensação de vazio na cabeça, palpitação, insônia e tremores enquanto manifestações clínicas do transtorno da ansiedade generalizada. Ao realizar-se a razão de chance, evidenciou-se 1,25 maior chance de mulheres não brancas apresentarem tremores em comparação com mulheres brancas. Mulheres sem parceria 
possuem 20,0\% menos chance $(\mathrm{OR}=0,792$ [IC=0,645-0,972]) de apresentarem tremores e mais de seis consultas pré-natais constitui-se fator de proteção para essa manifestação em 18,5\% (OR=0,815 [IC=0,681-0,975]) (dados não expostos em tabela).

Houve significância estatística entre número de consultas e nervosismo persistente $(\mathrm{p}=0,020)$. Mulheres com até cinco consultas de pré-natal possuem 98\% menos chance de possuírem nervosismo persistente $(\mathrm{OR}=0,019$ [IC=0,001-0,437]. Essa investigação evidenciou que a transpiração é duas vezes maior $(\mathrm{OR}=2,083$ [ $\mathrm{IC}=1,385-3,133])$ em mulheres não brancas, oito vezes maior $(\mathrm{OR}=8,000[\mathrm{IC}=1,522-42,042])$ entre aquelas com iniciação sexual antes dos 15 anos e 7 vezes maior $(\mathrm{OR}=7,313$ $[\mathrm{IC}=4,390-37,164])$ entre as participantes com menos de 20 semanas gestacionais, havendo associação significativa com sexarca $(\mathrm{p}=0,025)$ (Tabela 1$)$.

Tabela 1 - Razão de chance, aspectos sociais, reprodutivos e relacionados à gestação associados ao nervosismo persistente e transpiração. Baía da Traição, Paraíba, Brasil, 2019. (N=30).

\begin{tabular}{|c|c|c|c|c|c|c|c|c|}
\hline Variáveis & \multicolumn{4}{|c|}{ Nervosismo Persistente } & \multicolumn{4}{|c|}{ Transpiração } \\
\hline $18-25$ & 1 & 7 & 1,000 & 1,429 & 7 & 1 & 0,092 & 8,400 \\
\hline \multicolumn{9}{|c|}{ Renda (Salário mínimo) } \\
\hline Até 1 & 3 & 18 & 0,534 & 0,857 & 13 & 8 & 0,443 & 2,031 \\
\hline$\geq 2$ & 0 & 19 & & $(0,720-1,021)$ & 4 & 5 & & $(0,417-9,886)$ \\
\hline$\geq 10$ & 2 & 18 & & $(0,080-2,557)$ & 10 & 10 & & $(0,466-11,693)$ \\
\hline \multicolumn{9}{|l|}{ Etnia } \\
\hline Branca & 0 & 5 & 1,000 & 1,136 & 5 & 0 & $0,052 \ddagger$ & 2,083 \\
\hline Não branca & 3 & 22 & & $(0,983-1,313)$ & 12 & 13 & & $(1,385-3,133)$ \\
\hline \multicolumn{9}{|l|}{ Sexarca (anos) } \\
\hline Sem parceiro & 0 & 6 & & $(0,752-1,018)$ & 4 & 2 & & $(0,090-3,804)$ \\
\hline \multicolumn{9}{|l|}{ Planejada } \\
\hline Sim & 1 & 16 & 0,565 & 2,909 & 9 & 8 & 0,638 & 1,422 \\
\hline Não & 2 & 11 & & $(0,234-6,164)$ & 8 & 5 & & $(0,328-6,174)$ \\
\hline \multicolumn{9}{|l|}{ Consultas } \\
\hline $1-5$ & 1 & 26 & $0,020 \ddagger$ & 0,019 & 17 & 10 & 0,070 & 0,370 \\
\hline$\geq 6$ & 2 & 1 & & $(0,001-0,437)$ & 30 & 3 & & $(0,226-0,606)$ \\
\hline \multicolumn{9}{|c|}{$\begin{array}{l}\text { Idade Gestacional } \\
\text { (semanas) }\end{array}$} \\
\hline$<20$ & 1 & 16 & 0,565 & 0,344 & 13 & 4 & 0,250 & 7,313 \\
\hline$\geq 20$ & 2 & 11 & & $(0,028-4,273)$ & 4 & 9 & & $(4,390-37,164)$ \\
\hline
\end{tabular}

*significância estatística; †OR: Razão de Chance/IC: Intervalo de Confiança; \$Teste Exato de Fisher.

Fonte: Dados de Pesquisa.

Mulheres com maior renda possuem $19,0 \%(\mathrm{OR}=0,810)$ e sem parceiros possuem $16,7 \%(\mathrm{OR}=0,833)$ menos chance de apresentar tonturas. Além disso, houve associação estatística entre número de consultas e medo ( $p=0,030)$. Mulheres com seis ou mais consultas apresentam 74,1\% menor chance de apresentar medo ( $\mathrm{OR}=0,259[\mathrm{IC}=0,370-0,490])(\mathrm{Tabela} 2)$. 
Tabela 2 - Razão de chance, aspectos sociais, reprodutivos e relacionados à gestação associados tontura e medo. Baía da Traição, Paraíba, Brasil, 2019. (N=30).

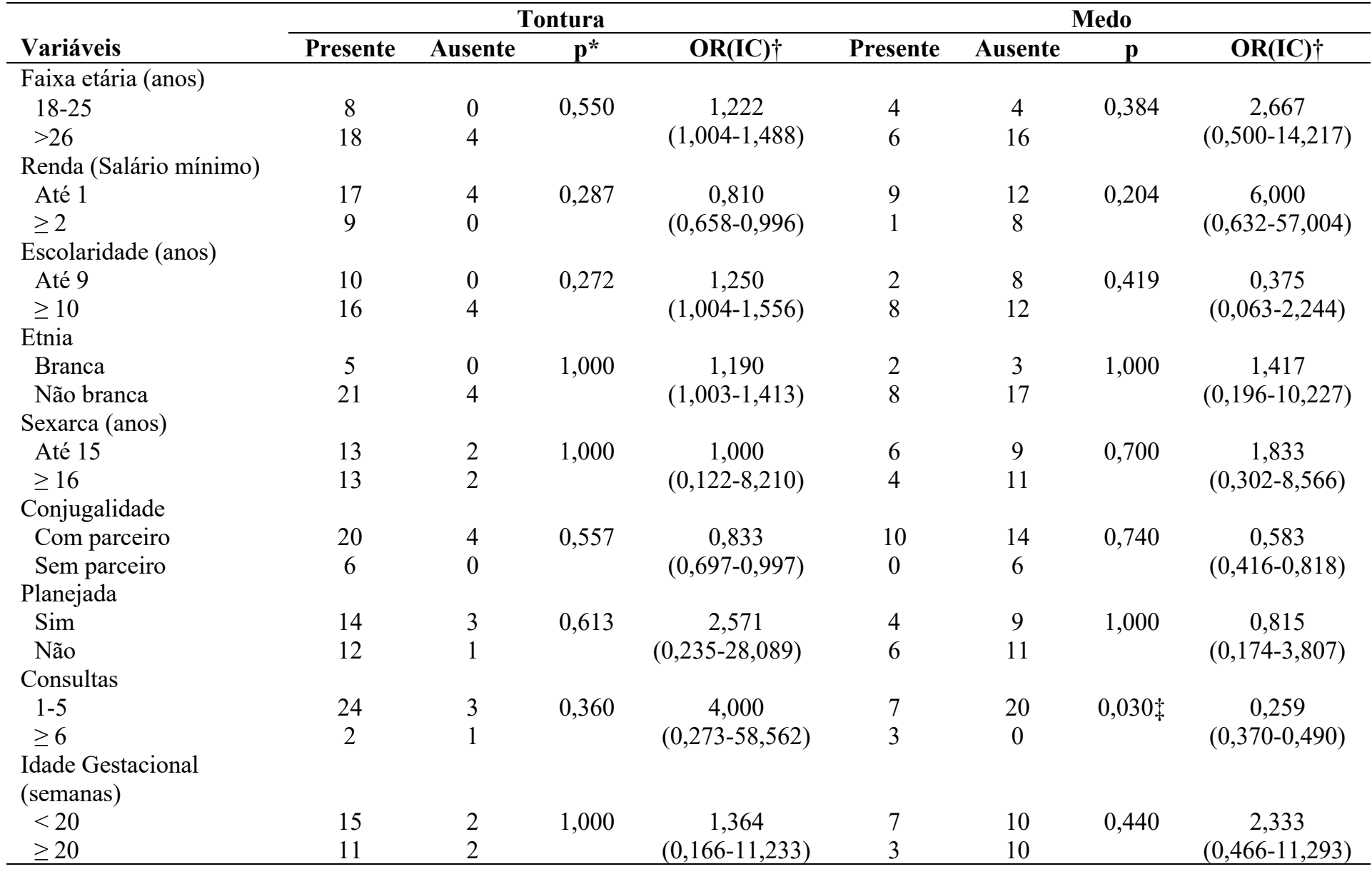

*significância estatística; †OR: Razão de Chance/IC: Intervalo de Confiança; †Teste Exato de Fisher. Fonte: Dados de Pesquisa.

$\mathrm{Na}$ Tabela 3, houve associação significativa entre etnia e inquietação ( $p=0,006)$, tensão muscular com escolaridade $(\mathrm{p}=0,011)$ e número de consultas $(\mathrm{p}=0,030)$. Gestantes não brancas apresentam 3,5 chances $(\mathrm{OR}=3,571)$ de manifestar inquietação em relação às brancas, assim como as mulheres que se submetem a menos de cinco consultas, apresentando $44,4 \%$ $(\mathrm{OR}=0,556)$ menor chance para demonstrar inquietação. Em relação à escolarização, aquelas com menos anos de estudo tinham duas vezes $(\mathrm{OR}=2,000)$ maior chance de apresentarem tensão muscular. Além disso, mulheres com iniciação sexual precoce possuíam oito vezes $(\mathrm{OR}=8,000)$ maior chance de apresentarem a tensão muscular como característica do transtorno da ansiedade generalizada. 
Tabela 3 - Razão de chance, aspectos sociais, reprodutivos e relacionados à gestação associados à inquietação e tensão muscular. Baía da Traição, Paraíba, Brasil, 2019. (N=30).

\begin{tabular}{|c|c|c|c|c|c|c|c|c|}
\hline \multirow[b]{2}{*}{ Variáveis } & \multicolumn{4}{|c|}{ Inquietação } & \multicolumn{4}{|c|}{ Tensão muscular } \\
\hline & Presente & Ausente & $\mathbf{p}^{*}$ & OR(IC) $\dagger$ & Presente & Ausente & $\mathbf{p}$ & OR(IC) $\dagger$ \\
\hline \multicolumn{9}{|c|}{ Faixa etária (anos) } \\
\hline $18-25$ & 6 & 2 & 0,410 & 2,500 & 5 & 3 & 1,000 & 0,778 \\
\hline$>26$ & 12 & 10 & & $(0,410-15,230)$ & 15 & 7 & & $(0,144-4,212)$ \\
\hline \multicolumn{9}{|c|}{ Renda (Salário mínimo) } \\
\hline Até 1 & 14 & 7 & 0,418 & 2,500 & 12 & 9 & 0,204 & 0,167 \\
\hline$\geq 2$ & 4 & 5 & & $(0,500-12,351)$ & 8 & 1 & & $(0,018-1,583)$ \\
\hline \multicolumn{9}{|c|}{ Escolaridade (anos) } \\
\hline Até 9 & 5 & 5 & 0,429 & 0,538 & 10 & 0 & $0,011 \ddagger$ & 2,000 \\
\hline$\geq 10$ & 13 & 7 & & $(0,115-2,519)$ & 10 & 10 & & $(1,290-3,100)$ \\
\hline \multicolumn{9}{|l|}{ Etnia } \\
\hline Branca & 0 & 5 & 0,006 & 3,571 & 4 & 1 & 0,640 & 2,250 \\
\hline Não branca & 18 & 7 & & $(1,905-6,696)$ & 16 & 9 & & $(0,217-23,324)$ \\
\hline \multicolumn{9}{|l|}{ Sexarca (anos) } \\
\hline Até 15 & 8 & 7 & 0,710 & 0,571 & 12 & 3 & 0,245 & 8,000 \\
\hline$\geq 16$ & 10 & 5 & & $(0,130-2,503)$ & 8 & 7 & & $(1,522-42,042)$ \\
\hline \multicolumn{9}{|l|}{ Conjugalidade } \\
\hline Com parceiro & 16 & 8 & 0,184 & 4,000 & 15 & 9 & 0,633 & 0,333 \\
\hline Sem parceiro & 2 & 4 & & $(0,600-26,683)$ & 5 & 1 & & $(0,033-3,327)$ \\
\hline \multicolumn{9}{|l|}{ Planejada } \\
\hline Sim & 11 & 6 & 0,547 & 0,636 & 11 & 6 & 1,000 & 1,227 \\
\hline Não & 7 & 6 & & $(0,145-2,784)$ & 9 & 4 & & $(0,263-5,734)$ \\
\hline \multicolumn{9}{|l|}{ Consultas } \\
\hline $1-5$ & 15 & 12 & 0,255 & 0,556 & 20 & 7 & $0,030 \%$ & 0,259 \\
\hline$\geq 6$ & 3 & - & & $(0,396-0,778)$ & - & 3 & & $(0,137-0,490)$ \\
\hline \multicolumn{9}{|c|}{$\begin{array}{l}\text { Idade Gestacional } \\
\text { (semanas) }\end{array}$} \\
\hline$<20$ & 8 & 9 & 0,141 & 0,267 & 12 & 5 & 0,602 & 1,500 \\
\hline$\geq 20$ & 10 & 3 & & $(0,054-1,326)$ & 8 & 5 & & $(0,325-4,905)$ \\
\hline
\end{tabular}

*significância estatística; †OR: Razão de Chance/IC: Intervalo de Confiança; †Teste Exato de Fisher. Fonte: Dados de Pesquisa.

$\mathrm{Na}$ Tabela 4, seguem as principais manifestações clínicas elucidadas pelas mulheres quanto ao Transtorno da Ansiedade Generalizada, onde 28 (20,0\%) mulheres eliciaram insônia, tendo como Diagnósticos de Enfermagem: sono comprometido e padrão de repouso alterado; 26 (18,6\%) relataram tontura e desconforto epigástrico e os Diagnósticos de Enfermagem foram: risco para ingestão de alimentos comprometida; $20(14,3 \%)$ indicaram tensão muscular, cujos Diagnósticos de Enfermagem foram: risco para movimento corporal comprometido e capacidade para gerir regime de exercício comprometida; 18 (12,9\%) relataram inquietação, tendo como Diagnósticos de Enfermagem: sono comprometido e padrão de repouso alterado. Além disso, 17 (12,1\%) entrevistadas sinalizaram transpiração, cujos Diagnósticos de Enfermagem foram: suor excessivo, padrão de higiene corporal comprometido, risco para baixa autoestima, risco para autoimagem negativa; 12 (8,5\%) mulheres referiram palpitações, gerando Diagnósticos de Enfermagem como: risco para ansiedade aumentada; 10 $(7,1 \%)$ relataram medos com Diagnósticos de Enfermagem: processo psicológico comprometido e risco de comportamento desorganizado. Menores frequências apontaram 5 (3,5\%) mulheres com tremores cujos Diagnósticos de Enfermagem foram: risco para movimento corporal aumentado e risco de capacidade para vestir-se/despir-se comprometida; 3 (2,1\%) apontaram nervosismo com Diagnósticos de Enfermagem: risco de confusão e risco de cognição comprometida e 1 (0,7\%) relatou sensação de vazio na cabeça com Diagnósticos de Enfermagem: risco para atenção comprometida e risco de capacidade para gerir regime medicamentoso comprometida. 
Tabela 4 - Manifestações clínicas do Transtorno da Ansiedade Generalizada e diagnóstico de enfermagem. Baía da Traição, Paraíba, Brasil, 2019.

\begin{tabular}{|c|c|c|c|}
\hline Variáveis* & f & $\%$ & Diagnósticos de Enfermagem \\
\hline Insônia & 28 & 20 & $\begin{array}{l}\text { Sono e repouso prejudicados; bem-estar prejudicado; Risco para atitude } \\
\text { familiar conflitante; }\end{array}$ \\
\hline Tontura e desconforto epigástrico & 26 & 18,6 & $\begin{array}{l}\text { Risco para apetite prejudicado; Risco para déficit no autocuidado para } \\
\text { alimentar-se; Risco para ingestão alimentar diminuída; }\end{array}$ \\
\hline Tensão muscular & 20 & 14,3 & $\begin{array}{l}\text { Movimento corporal diminuído; Falta de capacidade para gerenciar o regime } \\
\text { de atividades físicas; Risco para marcha descoordenada; }\end{array}$ \\
\hline Inquietação & 18 & 12,9 & Dificuldade para adormecer; Risco para sono e repouso prejudicados; \\
\hline Transpiração & 17 & 12,1 & $\begin{array}{l}\text { Transpiração excessiva; higiene corporal prejudicada; Risco para autoestima } \\
\text { alterada; }\end{array}$ \\
\hline Palpitações & 12 & 8,5 & Risco para ansiedade aumentada; Bem-estar prejudicado; \\
\hline Medos & 10 & 7,1 & $\begin{array}{l}\text { Bem-estar espiritual prejudicado; risco de comportamento desorganizado; } \\
\text { Risco para medo do parto; }\end{array}$ \\
\hline Tremores & 5 & 3,5 & $\begin{array}{l}\text { Nível de força dos membros diminuído; Risco para déficit de autocuidado } \\
\text { para vestir-se e despir-se; movimento corporal diminuído; }\end{array}$ \\
\hline Nervosismo & 3 & 2,1 & Risco de confusão; cognição prejudicada; \\
\hline Sensação de vazio na cabeça & 1 & 0,7 & $\begin{array}{l}\text { Risco para capacidade de autocuidado prejudicado; risco de confusão aguda; } \\
\text { capacidade de gerenciar o regime terapêutico diminuída. }\end{array}$ \\
\hline Total & 140 & 100 & 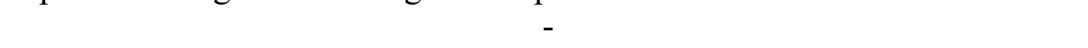 \\
\hline
\end{tabular}

*Variáveis de múltiplas respostas.

Fonte: Dados da pesquisa.

\section{Discussão}

A amostra pequena pode ter se constituído uma limitação da pesquisa. Os resultados deste inquérito populacional apontam que aspectos de caracterização da amostra constituem-se proteção ou risco às manifestações clínicas da ansiedade, aumentando a chance dos diagnósticos de enfermagem serem apontados na atenção primária, permitindo a criação de estratégias de apoio social e ajustamento de comportamentos voltados à redução dos riscos obstétricos e neonatais relacionados aos sinais e sintomas.

Corroborando a caracterização desse estudo, pesquisa com 3.936 gestantes holandesas identificou maioria com parceiro, primípara, não fumante e não etilista, com ensino médio completo. A gestação aumentou sete vezes o risco de ansiedade (OR: 7,95 [4,84; 13,05]), levando a qualidade de vida ruim (Bai, Raat, Jaddoe, Mautner \& Korfage, 2018). No Brasil, multigestas são mais ansiosas (Schiavo, Rodrigues \& Perosa, 2018).

Na perspectiva multidisciplinar, estudos nacionais indicam prevalência da ansiedade na gestação variáveis, sendo 13,6\% em Pelotas (Peter et al., 2017), 20,0\% em São Paulo (Costa, Souza, Pedroso \& Strufaldi, 2018), 26,8\% em Alfenas (Silva, Nogueira, Clapis \& Leite, 2017) e 64,9\% em São Luís (Morais, Simões, Rodrigues, Batista, Lamy, Carvalho, \& Ribeiro, 2017). Na Austrália, os sintomas de ansiedade são relativamente estáveis na gravidez, mas história anterior é um fator de risco na gestação atual (Schubert et al., 2017).

Nesse estudo, ausência de parceria e mais de seis consultas é fator de proteção aos tremores, não havendo explicação científica para o desfecho. Mulheres com menos consultas estavam menos nervosas. Hipoteticamente, menos consultas significa fornecer menos informações clínicas, o que pode refletir em pouca preocupação e menos nervosismo.

Com efeito, escores maiores de transtornos psicológicos e prejuízo na relação mãe/filho estão relacionados ao menor apoio social, situação economicamente desfavorável e ausência de companheiro no período gestacional (Cavalcante, Lamy Filho, França \& Lamy, 2017). Na Finlândia, as mães informaram mais sintomas ansiosos em comparação aos pais (Korja et al., 2018). Gestantes com pouco suporte possuem prejuízo na interação social afetiva, emocional, informativa e positiva. Logo, o 
apoio social percebido, reverbera diretamente no bem-estar materno e fetal, protegendo contra a ansiedade (Carvalho \& Benincasa, 2019).

O quadro de ansiedade não tratada durante a gravidez aumenta o risco de exposição ao uso de tabaco, álcool e outras drogas, além do risco de desnutrição e a dificuldade de seguir orientações médicas no pré-natal, diminuição da frequência às consultas, sendo associado ao risco da mortalidade neonatal (Souza, Santos, de Oliveira \& dos Santos, 2020). A ansiedade aumenta no terceiro trimestre, cuja ocorrência está associada à ocupação, ao histórico de abortamento, complicações gestacionais (Domingues, Fonseca, Leal, Aquino \& Menezes, 2020).

A transpiração pode ocorrer mais em mulheres não brancas, sexarca precoce e com menos de 20 semanas gestacionais. Dados sobre a associação da transpiração com as características étnicas e de sexualidade das gestantes não são bem elucidados, mas no primeiro trimestre as modificações fisiológicas da gestação podem estar relacionadas à transpiração.

A transpiração excessiva pode influenciar a autoestima e reverberar no estado emocional. Percepções sociais sobre o suor e odor aliado a higiene insatisfatória, pode levar ao isolamento social. Além disso, acredita-se na relação entre baixa escolaridade e conhecimento insatisfatório, geradores de dúvidas, medo e ansiedade. Talvez, a iniciação sexual precoce aliada à escassez de informação e a imaturidade provoquem aflição, quiçá aos sintomas de ansiedade. Desse modo, a consulta de prénatal proporciona informações em saúde relevantes nesse processo, evitando comportamentos autodepreciativos.

Sobre isso, estudo de avaliação da imagem corporal, autoestima e ansiedade realizada em Juiz de Fora com 386 gestantes concluiu que a ansiedade está ligada a uma imagem corporal negativa, acarretando atitudes corporais repulsivas (Meireles, Neves, Carvalho \& Ferreira, 2017). Mulheres não brancas ficam mais inquietas, sugerindo possível reflexo da condição socioeconômica e de vulnerabilidades sociais. Grávidas de etnia negra desenvolvem problemas específicos, em que a ocupação, condições de acesso aos serviços de saúde e demandas econômicas requerem planejamento singulares que favoreçam o acolhimento, humanização e integralidade do cuidado durante o pré-natal (Belfort, Kalckmann \& Batista, 2016).

Sobre diagnósticos de enfermagem com uso da CIPE, investigação em Vitória com 84 prontuários identificou ingestão de alimentos prejudicada, ingestão de líquidos baixa, risco para hipertensão, risco para hiperglicemia, edema nas pernas, dor pélvica e eliminação urinária aumentada (Primo et al., 2015), não havendo diagnósticos padrão de sono ou risco da ansiedade no processo gestacional. Embora especificidades clínicas sejam importantes, um olhar atento ao adoecimento mental na gestação favorece as pesquisas que utilizam o processo de Enfermagem como ferramenta do cuidado.

\section{Conclusão}

Houve relação do número de consulta pré-natal e nervosismo, transpiração com etnia e com mulheres com menos de 20 semanas gestacionais. Maior renda e ausência de parceria foram fatores de proteção à tontura e mais de seis consultas prénatais é fator de proteção ao medo. Atenção especial deve ser dada às mulheres não brancas, com idade superior a 26 anos. Os diagnósticos de enfermagem como sono comprometido e padrão de repouso alterado devem ser incorporados no plano de cuidados da atenção básica.

\section{Referências}

Bai, G., Raat, H., Jaddoe, V. W., Mautner, E., \& Korfage, I. J. (2018). Trajectories and predictors of women's health-related quality of life during pregnancy: A large longitudinal cohort study. PloS One, 13(4), e0194999. https://doi.org/10.1371/journal.pone.0194 999

Belfort, I. K. P., Kalckmann, S., \& Batista, L.E. (2016). Assistência ao parto de mulheres negras em um hospital do interior do Maranhão, Brasil. Saúde Soc, 25(3), 631-640. https://doi.org/10.1590/s0104-129020162571

Biaggio, A. M. B., \& Natalício, L. (2003). Inventário de ansiedade traço-estado-IDATE [State-Trait Anxiety Inventory-STAI]. Rio de Janeiro, Brazil: CEPA.

Carvalho, M. T., \& Benincasa, M. (2019). Depressão pós-parto e afetos predominantes na gestação, parto e pós-parto. Interação em Psicologia, 23(2). http://dx.doi.org/10.5380/psi.v23i02.57188 
Cavalcante, M. C. V., Lamy Filho, F., França, A. K. T. D. C., \& Lamy, Z. C. (2017). Relação mãe-filho e fatores associados: análise hierarquizada de base populacional em uma capital do Brasil-Estudo BRISA. Ciência \& Saúde Coletiva, 22, 1683-1693. https://doi.org/10.1590/1413-81232017225.21722015

Costa, D. O., Souza, F. I. S. D., Pedroso, G. C., \& Strufaldi, M. W. L. (2018). Mental disorders in pregnancy and newborn conditions: longitudinal study with pregnant women attended in primary care. Ciênc Saúde Coletiva, 23(3), 691-700. https://doi.org/10.1590/1413-81232018233.277 72015

Domingues, R. M. S. M., Fonseca, S. C., Leal, M. D. C., Aquino, E. M., \& Menezes, G. (2020). Aborto inseguro no Brasil: revisão sistemática da produção científica, 2008-2018. Cad Saúde Pública, 36, e00190418. https://doi.org/10.1590/0102-311X00190418

Fontoura, F. C., Cardoso, M. V. L. M. L., Rodrigues, S. E., Almeida, P. C. D., \& Carvalho, L. B. (2018). Anxiety of mothers of newborns with congenital malformations in the pre-and postnatal periods. Rev Latino-Am Enfermagem, 26, e3080. http://dx.doi.org/10.1590/1518-8345. 2482.3080

Korja, R., Nolvi, S., Kataja, E. L., Scheinin, N., Junttila, N., Lahtinen, H., Saarni, S., Karlsson, L., \& Karlsson, H. (2018). The courses of maternal and paternal depressive and anxiety symptoms during the prenatal period in the finn brain birth cohort study. PLoS One, 13(12), e0207856. https://doi.org/10.1371 /journal.pone.0207856

Meireles, J. F. F., Neves, C. M., Carvalho, P. H. B. D., \& Ferreira, M. E. C. (2017). Body image, eating attitudes, depressive symptoms, self-esteem and anxiety in pregnant women of Juiz de Fora, Minas Gerais, Brazil. Ciência \& Saúde Coletiva, 22(2), 437-445. https://doi.org/10.1590/141381232017222.23182015

Morais, A. O. D. D. S., Simões, V. M. F., Rodrigues, L. D. S., Batista, R. F. L., Lamy, Z. C., Carvalho, C. A. D., \& Ribeiro, M. R. C. (2017). Sintomas depressivos e de ansiedade maternos e prejuízos na relação mãe/filho em uma coorte pré-natal: uma abordagem com modelagem de equações estruturais. Cad Saúde Pública, 33(6), e00032016. https://doi.org/10.1590/0102-311X00032016

Nunes, J. T., Gomes, K. R. O., Rodrigues, M. T. P., \& Mascarenhas, M. D. M. (2016). Qualidade da assistência pré-natal no Brasil: revisão de artigos publicados de 2005 a 2015. Cad Saúde Coletiva, 24(2), 252-261. https://doi.org/10.1590/1414-462X201600020171

Organização Mundial da Saúde. (2017). Aumenta o número de pessoas com depressão no mundo. https://www.paho.org/bra/index.php?option=com content\&view=article\&id=5354:aumenta-o-numero-de-pessoas-com-depressao-no-mundo \&Itemid=839

Peter, P. J., de Mola, C. L., de Matos, M. B., Coelho, F. M., Pinheiro, K. A., da Silva, R. A., ... \& Quevedo, L. A. (2017). Association between perceived social support and anxiety in pregnant adolescents. Brazilian Journal of Psychiatry, 39(1), 21-27. http://dx.doi.org/10.1590/1516-4446-2015-1806

Primo, C. C., Trevizani, C. C., Tedesco, J. C., Leite, F. M. C., Almeida, M. V. S., \& Lima, E. D. F. A. (2015). Classificação internacional para a prática de enfermagem na assistência pré-natal. Enferm Foco, 6(4), 17-23. https://doi.org/10.21675/2357-707X.2015.v6.n1/4

Resende, F. Z., Almeida, M. V. D. S., Leite, F. M. C., Brandão, M. A. G., Cubas, M. R., Araújo, J. L., \& Primo, C. C. (2019). Terminological subset of the International Classification for Nursing practice (ICNP®) for breastfeeding support: content validation study. Acta Paul Enferm, 32(1), 35-45. https://doi.org/10.1590/1982-0194201900006

Schiavo, R. D. A., Rodrigues, O. M. P. R., \& Perosa, G. B. (2018). Variáveis associadas à ansiedade gestacional em primigestas e multigestas. Trends in Psychology, 26(4), 2091-2104. http://dx.doi.org/10.9788/TP2018.4-14Pt

Schubert, K. O., Air, T., Clark, S. R., Grzeskowiak, L. E., Miller, E., Dekker, G. A., \& Clifton, V. L. (2017). Trajectories of anxiety and health related quality of life during pregnancy. PloS one, 12(7), e0181149. https://doi.org/10.1371/journal.pone.0181149

Silva, M. M. D. J., Nogueira, D. A., Clapis, M. J., \& Leite, E. P. R. C. (2017). Anxiety in pregnancy: prevalence and associated factors. Rev Esc Enferm USP, 51, e03253. http://dx.doi.org/10.1590/S1980-220X2016048003253

Souza, V. M., Santos, R. S., de Oliveira, E. B., \& dos Santos, I. M. M. (2020). Mães adolescentes em consumo de álcool e outras drogas: estudo descritivo. Research, Society and Development, 9(8), e 774986359-e774986359. https://doi.org/10.33448/rsd-v9i8.6359

Triola, M. F. (2017). Introdução à estatística: atualização da tecnologia. In Introdução à estatística: atualização da tecnologia. LTC. 